\title{
METAFÍSICA Y EXISTENCIA: LA NOCIÓN DE LIBERTAD EN LA OBRA DE CORNELIO FABRO
}

\author{
Cristian Eduardo Benavides* \\ doi:10.11144/Javeriana.uph34-68.melf
}

\begin{abstract}
RESUMEN
En el presente trabajo se abordan algunos de los conceptos y principios más importantes de la obra de Cornelio Fabro, con el fin de ponderar el alcance, originalidad y consistencia de su posición especulativa respecto de la noción de libertad. En la primera parte, se presenta el itinerario intelectual y científico de Cornelio Fabro. En la segunda, se examina el fundamento metafísico y existencial de la libertad y se hace un recorrido filosófico por los autores más representativos de la modernidad que intervienen en su pensamiento. En la tercera, se realizan algunas consideraciones finales que delimitan los puntos centrales desarrollados en el trabajo.

Palabras clave: ser; libertad; metafísica; existencia; Cornelio Fabro
\end{abstract}

* Universidad Nacional de Cuyo, Mendoza, Argentina

Correo electrónico: cristianeduardobenavides@yahoo.com.ar

Para citar este artículo: BenAVIDES, C.E. (2017). Metafísica y existencia: la noción de libertad en la obra de Cornelio Fabro. Universitas Philosophica, 34(68), pp. 81-114. ISSN 0120-5323, ISSN en línea 2346-2426. doi:10.11144/Javeriana.uph34-68.melf 


\title{
METAPHYSICS AND EXISTENCE: FREEDOM NOTION IN CORNELIO FABROS'S WORK
}

\author{
Cristian Eduardo Benavides
}

\begin{abstract}
In the present paper, some of the most important concepts and principles of Cornelio Fabro's work are addressed, in order to weigh the scope, originality and consistency of his speculative position on the notion of freedom. In the first part, the intellectual and scientific itinerary of Cornelio Fabro is presented. In the second part, the metaphysical and existential ground of freedom is examined and a fast tour around the most representative philosophical authors of modernity involved in his thinking is done. In the third part, some brief final considerations are made in which the central topics developed in this work are delimited.
\end{abstract}

Key words: being; freedom; metaphysics; existence; Cornelio Fabro 


\section{Introducción}

Cornelio Fabro eXamina la noción de libertad desde ángulos y puntos de vista muy diversos, lo que demanda al investigador una lectura cuidadosa y atenta para no confundir la perspectiva filosófica desde la cual se abordan los conceptos y temas en concreto. En sus escritos se analizan y toman en consideración múltiples pensadores de diferentes períodos históricos. Por mencionar algunos: Parménides, Platón, Aristóteles, los pensadores neoplatónicos, San Agustín, San Buenaventura, Descartes, Hume, Kant, Fichte, Hegel, Feuerbach, Husserl, Jaspers, Heidegger. Entre todos ellos, en los trabajos del filósofo italiano, sobresalen dos autores: Tomás de Aquino y Søren Kierkegaard ${ }^{1}$.

En líneas generales, el pensamiento de Cornelio Fabro es reconocido dentro del marco filosófico del tomismo del siglo XX. En efecto, el filósofo italiano procura desentrañar y, a la vez recuperar, apartándose de las interpretaciones escolásticas prevalecientes en su época, la esencia y la originalidad especulativa de la metafísica del doctor medieval ${ }^{2}$. Sin embargo, el diálogo, la confrontación y la discusión permanente con otros autores, en especial con aquellos del mundo moderno y contemporáneo, conducen a Fabro a una singular exposición y tratamiento de los temas ${ }^{3}$.

Por tal motivo, puede afirmarse que no resulta correcto ubicar a Fabro -sin hacer las precisiones correspondientes- dentro de la escuela "Neotomista", pues dicho rótulo deja de lado la multitud de escritos y traducciones que realiza de autores que no se vinculan directa ni indirectamente con la obra de Tomás de

1 "Fabro è noto agli studiosi soprattutto per i suoi contributi storiografici fondamentali alla comprensione di san Tommaso d'Aquino e di Soren Kierkegaard" (De Anna, 2012b, p. 9).

2 "A metafisica tomista no século XX encontra em Cornelio Fabro sua figura mais expressiva. Em diálogo com os mais importantes filósofos do pensamento ocidental, e através de rigoroso itinerário, Fabro recuperou a originalidade do pensamento de Santo Tomás, a despeito das sombras lançadas ao longo da história sobre o tomismo, especialmente em sua própria escola" (Calvet da Silveira, 2006, p. 1).

3 "La ricca riflessione teoretica di Cornelio Fabro corre parallela alla sua ricostruzione della storia del pensiero moderno. In un confronto assiduo, non usuale nei filosofi di secolo tomista, con la problematica moderna, egli si è lasciato interrogare in profondita da essa reagendo criticamente e, al contempo, accogliendone suggestioni e prospettive tutt'altro che marginali" (Borghessi, 2012, p. 105).

4 "Fabro non si lo poteva ricondurre sotto nessuna etichetta, nemmeno sotto quella di tomista, pur essendo uno dei massimi studiosi e interpreti che l'Aquinate abbia avuto nel Novecento" (Pizzuti, 2000, p. 11). 
Aquino. Es oportuno recordar aquí las traducciones del alemán y del danés, precedidas por extensas introducciones y notas, que el filósofo italiano hace de las obras de Marx, Engels, Feuerbach, Hegel, Adler y Kierkegaard. Una señal inequívoca de la importancia y de la envergadura de dicha empresa es el hecho de que la edición italiana de la obra de Kierkegaard supere hasta el momento de la muerte de Fabro, año 1995, a la misma edición crítica danesa ${ }^{5}$. Más allá de que el filósofo italiano sea considerado, con cierta justificación, uno de los principales estudiosos del dominico durante siglo pasado, es menester señalar que los temas que desarrolla, los pensadores que aborda y la forma misma con la que articula tales temas y autores tornan dificultosa una clasificación de su pensamiento ${ }^{6}$.

Siguiendo a Goglia (2010, p. 228), Fabro escribió, en su larga trayectoria intelectual, cerca de 40 libros, alrededor de 50 cuadernillos de clase y casi 900 artículos, sin contar las traducciones íntegras o parciales de 20 obras de los autores antes citados. Se trata de una producción científica realmente voluminosa que puede resumirse, a muy grandes rasgos, en tres ejes principales de investigación ${ }^{7}$ :

a) La profundización de la noción metafísica de participación.

b) La determinación del principio moderno de inmanencia.

c) El replanteamiento de los conceptos y argumentos de corte existencialista.

5 En los años cuarenta Fabro comienza una tarea ininterrumpida que lo consagra rápidamente, como declara Nicola Abbagnano (1990, p. 63), en el máximo exégeta en Italia del pensador danés. Para tomar noticia de algunos aspectos específicos de la traducción e interpretación de Fabro y de la difusión y recepción del pensador danés en Italia puede leerse a Rizzacasa (1997, pp. 261-283) y Davini (2004, pp. 356-369).

6 "Si può dire che Fabro è tomista e non è tomista. Certo non è neo-tomista di scuola, benché abbia imparato dai Domenicani. Ma in relazione a san Tommaso, possiamo dire che Fabro fa una lettura di quelli che egli considera i testi principali dello stesso san Tommaso, che non sempre è d'accordo con tutti i testi dell'Angelico. Forse si potrebbe sostenere che Fabro sia un tomista post-kantiano, post-hegeliano, post-kierkegaardiano, oppure, alla rovescia, un kierkegaardiano tomista" (Sánchez Sorondo, 2007, p. 44).

7 Jörgen Vijgen recoge a partir de las investigaciones realizadas por los estudiosos del Progetto Culturale Cornelio Fabro algunos de los siguientes leitmotivs: "1) The centrality of the metaphysics of participation; 2) the ontological importance of the actus essendi; 3) his critique of any formalistic essentialist; 4) the dialogue and confrontation with modern philosophy; 5) the importance of a thomistic epistemology; 6) the "principle of immanence" which lies at the heart of modern atheism and liberal theology; 7) his retrieval of Kierkegaard; 8) the contribution of thomism towards a genuine understanding of freedom; 9) his insistence on a return towards metaphysics" (Vijgen, 2005, p. 199). En opinión de quien escribe, tales puntos bien pueden sintetizarse en los tres mencionados. 
Tales indagaciones presentan, como telón de fondo, dos temáticas fundamentales de todo su pensamiento filosófico: la cuestión del ser y la cuestión de la libertad (Dalledonne, 2001).

Fabro considera que la doctrina de Tomás de Aquino ofrece, en virtud de su profunda concepción del ser y de la participación, un sólido fundamento metafísico para la comprensión del concepto de libertad. No obstante lo mencionado, opina que en ciertos pasajes de su obra el dominico acentúa demasiado los aspectos estáticos del obrar humano, dejando un tanto oscurecida la dimensión existencial. Este hecho, Tomás de Aquino lo induce a la lectura y estudio de algunos filósofos modernos, y principalmente a la de Kierkegaard, cuyos escritos le brindan una penetrante reflexión de la subjetividad que le permiten -desde su propio pensamiento- ahondar en aquellos puntos que no halla tratados a fondo en la obra del Aquinate ${ }^{8}$. La peculiar interpretación de la libertad que presenta Fabro, sustentada en dichas premisas, tiene como resultado una serie de proposiciones, razonamientos y tesis que, lejos de ser aceptadas sin cuestionamiento, son materia de discusión entre los expertos? ${ }^{9}$. En general la crítica que se alza contra el filósofo italiano gira en torno a los tres puntos siguientes:

a) El abandono de los principios que formula Tomás de Aquino.

b) La afinidad o cercanía especulativa que asienta en determinados temas entre el doctor medieval y los filósofos modernos.

8 María José Binetti nota, en esta dirección, que el estudio del filósofo italiano ilumina ciertas zonas de posible convergencia entre el Aquinate y el existencialista danés: "En breve: Fabro asentó la metafísica espiritual de Kierkegaard sobre una metafísica del ser como acto intensivo emergente que no es propia, pero quizá tampoco fundamentalmente refractaria al pensador danés. De más está decir que Kierkegaard desconoce el esse ut actus de Santo Tomás. Sin embargo, según Fabro, el danés reconocería, en el mejor sentido aristotélico, la primacía del acto sobre la potencia, la diferencia entre el ámbito empírico y el real, entre el metafísico y el fenomenológico. Por otra parte, la libertad originaria de Kierkegaard podría coincidir con la voluntas ut natura de Tomás de Aquino" (Binetti, 2003, p. 102).

9 Comenta Abelardo Lobato (1996) sobre este asunto: "Con este pensador [Kierkegaard] Fabro quedó fascinado. Comprendió la novedad del problema de la libertad, la fuerza que tiene en la modernidad, y trató de darle respuesta. Para esto también recurrió a Tomás de Aquino. La fortuna no le sonrió tanto en este empeño. Tomás reconoce la libertad del hombre, como esencial al acto humano, pero no la distancia de la racionalidad, ni de la inteligibilidad. Fabro buceó en todos los textos tomistas. Soñó a veces con haber encontrado la primacía de la libertad en Tomás: Intelligo quia volo! Pero aquí no ha tenido el consenso de los especialistas. Esa es una cuestión abierta" (p. 53). 
c) La fuerte influencia que ejerce en sus escritos el pensamiento de Kierkegaard.

Una vez esbozado el itinerario científico e intelectual de Cornelio Fabro, a continuación se aborda el desarrollo especulativo de los temas capitales de su obra.

\section{Entre el ser y la libertad}

De ACUERdo CON LA INTERPRETACión de Fabro, el ser en cuanto tal, esto es, considerado en sí mismo, precede a toda oposición categorial: no es forma ni contenido, ni sujeto ni predicado, ni yo ni no-yo, ni esencia ni existencia, ni objeto ni sujeto $^{10}$. Tales términos señalan una "caída ontológica" del ser en otra cosa que él mismo, pues no designan el ser sin agregados, sino el ser bajo una forma determinada. El ser en cuanto ser, empero, no dice ser esto o aquello, de este modo o de aquel otro, sino que carece, como tal, de toda determinación y particularidad ${ }^{11}$.

Solo el ser ostenta así la prerrogativa de comparecer ante la conciencia como momento primigenio -anterior a toda duda y exento de todo error- que es inicio o punto de partida (Anfangspunkt) del pensamiento. Para Fabro (1969a, p. 244) esta exaltación del ser como prius absoluto no solo se halla en la obra de Tomás de Aquino, sino que aparece particularmente ejemplar en la Modernidad con la obra de Hegel, sea por la clara conciencia resolutiva que trae al problema especulativo en su devenir histórico, sea, y sobre todo, por la recuperación del ser (Sein) en el centro de la dialéctica, como inicio, medio y término de la misma.

10 De aquí brota la bella expresión de Fabro (1965): "Nellessere perciò non si entra, perché in esso si è sempre, e dall'essere il pensiero non esce perché uscire dall'essere è per il pensiero uscire da se stesso, e questo è per la coscienza contradittorio: sarebbe un essere cosciente ch'è a un tempo non-essere cosciente ovvero un 'decidere' di abbandonare se stessi perportarsi "al di là" dell'essere. Ma un "al di là dell'essere" non si può dare, non piu di un al di qua dell'essere stesso. E cosa sarebbe mai un portarsi al di qua o al di là dell'essere?" ( p. 12). Cabe señalar que aún contando con los tomos publicados hasta el momento de la edición crítica de las Opere Complete del filósofo italiano que lleva adelante el Progetto Culturale Cornelio Fabro, en el presente artículo se cita -siempre que se pueda y por cuestiones estrictamente metodológicas- los originales según sus primeras ediciones, a no ser que las posteriores hayan sido corregidas o ampliadas por el mismo Fabro, en cuyo caso se utilizarán preferiblemente.

11 Por esta razón, Fabro declara que la existencia del ente finito es un "dato de hecho", y no un contenido nocional alcanzado mediante el análisis, que tiene su fundamento último en el profundo misterio de la libertad divina que es el Ser sin agregado (Fabro, 1963d, pp. 330-334). 
A pesar de la convergencia que plantea Fabro entre el Aquinate y Hegel en lo que se refiere a la problemática filosófica de fondo, estima que la posición metafísica de ambos autores se revela, entre sí, sustancialmente divergente. Se trata, según una expresión técnica propia del filósofo italiano, de una "correspondencia antitética”, fórmula que indica a la vez la semejanza de método y la desemejanza de contenido que se evidencia entre tales instancias. Dos cuestiones cardinales, relacionadas íntimamente con la problemática del ser dan cuentas de lo señalado: por una parte, la inmanencia metafísica de lo Infinito en lo finito; por otra parte, la positividad ontológica de la nada ${ }^{12}$.

En cuanto al primer asunto, es menester advertir que los dos autores cumplen -de manera análoga- la exigencia parmenídea de la unidad del ser, pues ambos sostienen que lo finito, en sí mismo considerado, no agrega nada al Ser. No obstante, se hace patente la diferencia en el modo de entender la relación finito-Infinito, pues mientras que Hegel -según interpreta Fabro- concibe la pluralidad de los entes como simple aparición (Erscheinung) dentro de la unidad del concepto, el dominico afirma una doble Diremtion: por un lado, en el origen, en virtud del proceso de la creación del ser finito por parte de Dios; por otro lado, en la constitución, en razón de la "composición" metafísica del ser finito en el horizonte de la participación ${ }^{13}$.

En la obra de Tomás de Aquino la presencia de Dios es el fundamento tanto de la positio metafísica de todos los entes cuanto de su actuar, puesto que los mismos son concebidos como causas creadas. En el sistema hegeliano, en cambio, la presencia del Absoluto absorbe la finitud, puesto que el "Infinito positivo" - no el "Infinito negativo" que es más bien un Otro que el finito, y por ende, alteridad

12 Para Fabro, los dos autores mencionados representan -junto con el Estagirita- los más insignes metafísicos de la filosofía occidental: "Ciò che Aristotele ha significato nellantichità e S. Tommaso nel Medio Evo per la risoluzione della problematica del loro tempo, questo Hegel rappresenta nell'età moderna: ognuno di essi ha saputo, nella propria epoca, portare l'occhio piu a fondo e piu avanti; $\dot{e}$ riuscito cioè a collocarsi sul vertice della vita dello spirito dove si dileguano le opposte determinazioni per attingere all'affermazione dell'Assoluto che veramente è coincidenza di Essere e Vita, di Essere e Perfezione, di Essere e Pensiero: "l'Essere compiuto", come dice Hegel" (Fabro, 1983d, p. 5).

13 "Essi si muovono, apparentemente, spinti dalle medesime istanze di conciliazione fra filosofia e religione, fra ragione e fede... per risolvere il rapporto di finito-Infinito, di uomo-Dio..., ma divergono nel fondo e nel profondo, nel significato e nel segno cioè dell'essere, nell'antitesi dell'immanenza e della trascendenza che non ammette conciliazione ma traduce una tensione infinita" (Fabro, 1969c, pp. 599-600). 
no superada- se revela, en última instancia, como la unidad dialéctica y sin residuos de finito e Infinito ${ }^{14}$. Toda realidad finita queda disuelta en el no-ser, o mejor dicho, en el no-ser "en sí", en un momento de la manifestación de lo Absoluto. De allí que Fabro repute que la "suficiencia" del Ser Divino que Hegel defiende en su sistema -más allá de que coincida con Tomás respecto de la indivisible unidad del Ser en Dios- traiga a la par una idea radicalmente distinta, a saber, la "insuficiencia" del ser finito ${ }^{15}$.

En cuanto al segundo asunto, ambos autores estiman que el ser inicial y finito que se presenta de forma inmediata a la conciencia revela en sí mismo, en su incesante desaparecer, la presencia del no-ser. La discordancia, sin embargo, se hace patente en lo que respecta a la valencia que cada uno concede a la noción de noser. Mientras que Hegel declara que entre el ser y la nada hay un intercambio de identidad ${ }^{16}$, el dominico considera que la nada tiene un significado real. Para el filósofo alemán, ser y no-ser se encuentran comprendidos -según una perfecta e inescindible unidad- en el interior mismo del concepto de "devenir", dentro del cual desaparece toda oposición. Para Fabro (1963c, p. 123), más allá de toda tensión monista entre ser y no-ser, Sto. Tomás, por su parte, concibe a la nada como una noción intermedia entre el Ser y el ente, entre Dios y las creaturas, entre el Uno y los muchos. La nada delata el no-ser que la creatura es sin el acto creador de Dios. Por tal razón, debe hablarse de la nada de forma positiva, es decir, como una

14 "Tutta la filosofia di Hegel -ch'è ad un tempo di massima semplicità e di estrema complessità- si presenta come un movimento alterno di "trasferimento" del finito nell'Infinito e dell'Infinito nel finito: il finito si trasferisce nell'Infinito come nella sua realtà e l'Infinito si trasferisce nel finito come sua manifestazione" (Fabro, 1968a, pp. 39-40).

15 Escribe Fabro (1969a): "Quel che preme ad Hegel di mantenere, con la Bibbia, è l'assoluta indivisibile unità dell'Essere in Dio -l'esse intensivo = das erfülltes Sein- nella forma della "vita" perfetta del Nous supremo aristotelico. In questo senso, se si può dire, Hegel è d'accordo con $S$. Tommaso nel mantenere l'assoluta immutabilità di Dio, anche durante e dopo la creazione e l'Incarnazione: Dio non può avere alcuna relazione reale con le creature, Dio nulla ha acquistato per sé con la creazione e l'Incarnazione. Questa sufficienza assoluta di Dio è da Hegel sollevata alla più intensa formula speculativa, e in questa forma, potrebbe rientrare nella filosofia cristiana. Se non che la sufficienza della attualità di Dio è fatta da Hegel solidale con l'insufficienza e l'irrealtà-idealità-del finito" (p. 39).

16 "The Hegelian nothingness is the pure negative which is identical with empty and abstract being; it is dissolved in a logical moment which is consequent on being-finite being-. Nothingness ends up by not being nothingness except as a mere dialectical moment" (Fabro, 1969a, p. 159). 
realidad ontológicamente diferente del ser, que expresa de suyo tanto la indigencia absoluta de la creatura cuanto la infinita potencia del Creador ${ }^{17}$.

La cuestión decisiva que subyace a toda esta problemática, según Fabro, es concretamente la referente a la determinación del cominciamento filosófico. Para Hegel la filosofía tiene como inicio o comienzo, propiamente dicho, la noción de $\operatorname{ser}^{18}$. En efecto, el ser se presenta al pensamiento como lo más inmediato, universal e indeterminado. Ahora bien, por la misma razón que el ser se descubre como punto de partida se manifiesta también -en esta primera instancia- como la noción más abstracta, simple y pobre del saber. De allí que en el sistema hegeliano el problema del cominciamento se resuelva, puntualmente, en un pasaje dialéctico desde el Ser vacío inicial (leeres Sein) al Ser pleno terminal (erfülltes Sein) ${ }^{19}$. Para Tomás de Aquino el esse del comienzo, por el contrario, no es un ser abstracto sino máximamente concreto, pues entendido en su forma gramatical de participio (ens) designa la síntesis de nombre y verbo, contenido y forma, sujeto y acto ${ }^{20}$.

El ens, indica el filósofo italiano, contiene a la vez y sin contradicción, la mayor indeterminación y determinación que atañe en sí al principio. Es completamente indeterminado en la línea formal del contenido porque no indica ninguna

17 "Per il Cristianesimo e la metafisica creazionistica invece il non essere è il "nulla" che si trova fra l'Essere - ch'è Uno $=$ sussistente $=$ Dio- el'essente - che sono $i$ molti $=$ la creatura $-:$ questo nulla non è $i l$ semplice non essere, come generalizzazione della formula negativa della copula, né il non essere secondo che si attesta nella posizione dell'esperienza sempre mutevole delle sue presentazioni e coll'alternarsi dei processi di generazione e corruzione nel sempre vario aspetto del mondo. Il nulla dal quale Dio col suo atto creativo toglie la creatura è precisamente quel che la creatura è, il quel-che-non-è, prima efuori dell'atto creativo di Dio" (Fabro, 1961, p. 20).

18 En cuanto a la interpretación fabriana del comienzo, con particular referencia a la Ciencia de la Lógica de Hegel, véanse: Knappik, 2012. pp. 269-287 y Negro, 2009, pp. 363-380.

19 "Tutto l'affannarsi di Hegel attorno al cominciamento si risolve in un "passaggio" all'interno del concetto ossia nel passaggio dal concetto iniziale di essere del tutto vuoto e indeterminato al concetto finale ch'è il Tutto onnicomprensivo e onnipresente, l'essere compiuto intensivo ch'è Dio stesso e questo avviene per Hegel mediante lo stesso e identico termine "essere" -Sein-" (Fabro, 1969a, p. 370).

20 No obstante los límites que Fabro encuentra en el pensamiento de Hegel, no deja de subrayar la importancia y valor excepcional de su obra: "Quel che a me interessa in modo supremo nell'opera di Hegel ormai da alcuni decenni e che ora dopo l'opera di Heidegger mi stimola senza darmi pace, è di chiarire cosa intende Hegel per "Essere"-Sein-, cosa deve intendere nel primo semantema di "essere", come la filosofia a partire dall'essere può porre e fare il primo passo del pensiero che cerca di fondare se stesso. So bene che la filosofia contemporanea di ogni tendenza trova questi problema senza senso: ma io sono convinto invece che sono ancora gli unici problemi che meritano la nostra riflessione, se non si vuol lasciare senza senso il senso della stessa vita e della libertà" (Fabro, 2004, pp. 63-64). 
esencia en particular, pero también es completamente determinado en la línea del acto porque señala el ejercicio -en acto- del acto de ser. De esta manera, la verdad del Ser terminal (Ipsum Esse) es asegurada y mantenida por la verdad misma del ser inicial (ens) $)^{21}$.

El principio de los diferentes análisis especulativos es el de participación, noción que -según Fabro- expresa el punto último de referencia metafísico de la creatura, tanto desde el punto de vista estático de su estructura como desde el dinámico de su dependencia causal de Dios (Fabro, 1969a, p. 435). En cuanto a la constitución metafísica del ente finito, debe apreciarse que la distinción "real" -y no solo de razón- entre la esencia y el ser aparece en la obra de Tomás de Aquino como una tesis central, que se erige, efectivamente, sobre la base teorética de la noción de participación ${ }^{22}$. El esse se predica de Dios de modo esencial, en tanto que se predica de las creaturas por participación. En efecto, no puede decirse de ninguna creatura que su esencia o substancia sea su ser sino, solamente, que tiene ser. Pues bien, todo lo que es tal por participación se divide, necesariamente, en participante y participado, potencia y acto. Como afirma Fabro, lo participante es la essentia o suppositum; lo participado, el esse o actus essendi ${ }^{23}$.

En sentido estricto, el término ens indica el ejercicio de la formalidad de ser. Ente es lo que es (id quod est) o bien, lo que tiene ser (id quod habet esse). Siendo, por lo tanto, aquello que recibe el ser, que posee el ser, pero que no se identifica con el ser; así, se denomina ente, de acuerdo con la declaración del Aquinate, a

21 Para una visión global de la posición que adopta el filósofo italiano sobre este tema en particular, véase: Olivera, 2007.

22 "La distinzione reale pertanto fra essenza ed esse nelle creature va riconosciuta come la verità centrale della metafisica, mentre la nozione di partecipazione è la verità fondamentale che comanda quella tesi capitale" (Fabro, 1969, p. 369). Mondin considera, a diferencia de lo que opina Fabro, que la clave del pensamiento de Tomás de Aquino se encuentra propiamente en la noción de esse intensivo: "A dire di molti studiosi autorevolissimi -Gilson, Masnovo, Maritain, Fabro ecc. - la dottrina della distinzione reale tra essenza e atto d'essere rappresenta uno dei grandi cardini, anzi il cardine principale di tutta la costruzione filosofica di $S$. Tommaso. A noi pare che il vero cardine di tutto l'edificio tomistico sia non la distinzione reale, bensi l'essere concepito intensivamente" (Mondin, 2000, p. 261). Para un estudio más detenido sobre este tema, véanse: Wippel (2012, pp. 139-156) y Pandolf (2006, pp. 137-152).

23 "La sintesi tomista è assolutamente originale: essa infatti accoglie il nucleo metafisico della dottrina della trascendenza platonica [...], che viene saldato con l'atto dell'immanenza aristotelica" (Fabro, 1960a, p. 41). 
aquello que participa de forma finita del $\operatorname{ser}^{24}$. Considerado en su absoluta pureza, el esse no dice más que ser. Sin embargo, lejos de manifestarse subsistente y acabado, se revela en los entes como un acto inherente, imperfecto y limitado. Sin importar su magis et minus de perfección, el ente siempre se muestra en relación con Dios -identidad plena de ser y esencia- como un ser degradado, cuya naturaleza hace ostensible la restricción y confinamiento del esse a un modo determinado de ser. Así, el ente denota lo real que ha superado tanto el abismo vacío de la nada como la multiplicidad vacía categorial de lo meramente posible. Empero, paga su afirmación de realidad al duro precio de no ser el esse $e^{25}$.

Ha de tenerse en cuenta, en el desarrollo de este tema, que el término esse tiene para Fabro un significado distinto de aquel que conlleva el término existentia. El primero designa el ser como actus; el segundo, el ser como factum y, por ende, como realidad contingente y extrínseca ${ }^{26}$. La equivalencia semántica de tales términos tiene como correlato la flexión o sustitución de la distinción-composición de la pareja esse-essentia como co-principios internos reales del ente, por la unificación-reducción de los mismos a dos simples aspectos de una sola realidad: la de la esencia en su estado de posibilidad (essentia), o la de la esencia en su estado de realidad o realización efectiva (existentia). Para el filósofo italiano, el esse es concretamente el acto que presentifica la presencia de la existencia; lo que significa,

24 "Ens autem dicitur id quod finite participat esse" (Tomás de Aquino, In Librum De Causis, lect. 6).

25 Para Fabro, explica Villagrasa, el método propio de la metafísica de Tomás de Aquino es el de la reductio o resolutio. Esta reductio no es un procedimiento meramente lógico, procedente de análisis y razonamientos, sino una fundación en los principios, un movimiento sintético opuesto a la división y composición. La resolutio va de los efectos a la causa, de lo particular a lo universal, de los actos múltiples y superficiales al acto más común, primero y último (Villagrasa, 2011, pp. 416-418).

26 "Questo esse, cosi preclaro, non può essere la existentia della tradizione scolastica ch'è passata nel razionalismo moderno e di cui la scolastica è in buona parte responsabile: tale existentia [...] è il fatto di essere e percio il risultato della attuazione fisica e metafisica degli esseri quale può essere individuata nelle coordinate di spazio e tempo. Tale existentia è estranea alla metafisica tomista e sta agli antipodi dello esse atto ultimo metafisico, qual è stato prospettato da $S$. Tommaso al vertice della dialettica dei gradi e della partecipazione. Infatti esso è nascosto nel segreto del reale e mentre è l'atto della sostanza, non si fa presente per suo conto ma mediante l'attualità della sostanza stessa e delle sue modificazioni" (Fabro, 1969, p. 377). 
invirtiendo los términos, que la existencia no es sino el "resultado" del esse en su venir a $\operatorname{ser}^{27}$.

Atendiendo a los principios metafísicos señalados, Fabro nota que la creatura no solo se revela como ens per participationem sino también como agens per participationem. Queda así en evidencia la simetría que se establece entre las diferentes esferas u órdenes. En efecto, las potencias que en el orden dinámico se presentan como principio propio e inmediato del obrar presuponen en el orden estático la potencia intrínseca de la esencia. A su vez, los actos que en el orden dinámico se presentan como perfección de la virtus operativa presuponen en el orden estático el acto de ser, de modo que el acto segundo presupone el acto primero (Fabro, 1961, pp. 372-373).

Sin negar la causalidad y producción primera del esse por parte de Dios, el filósofo italiano destaca en el ámbito del fieri una causalidad segunda de la creatura sobre el ser. La actividad del ente finito, efectivamente, no se agota en una relación extrínseca de dependencia de la Causa universal, sino que se funda en el propio actus essendi del cual participa y tiene como término la divina causalidad ${ }^{28}$.

Este asunto abre paso a la cuestión acerca del influjo de la Causa primera sobre la voluntad creada en lo relativo al acto de elección. Así como en el orden estático, observa Fabro, se puede apreciar -según una distinción analítica- un primer momento de creación y conservación del esse y un segundo momento de especificación propio de las formalidades particulares que le suceden; así tambien, en el orden dinámico se puede apreciar un primer momento de puesta en acto tendencial, es decir, un movimiento primero hacia el bien por parte de la voluntad, y un segundo momento de elección particular en la esfera concreta de la existencia (Pangallo, 1991, pp. 109-122).

Con respecto al primer momento, hay que advertir que Dios no solo se presenta como causa del ser, sino también como causa del obrar de todos los agentes. En efecto, la voluntad creada -dada su finitud ontológica- se encuentra

27 La dupla abstracta de esencia-existencia no solo ha sido funesta para la escolástica y el tomismo sino incluso para el mismo pensamiento moderno, el cual con el cogito se terminó dirigiendo, según interpreta Fabro (1997, pp. 271-272), hacia un callejón sin salida.

28 Con respecto a la justificación y fundamentación del principio de causalidad en el principio de participación, véanse: Pellecchia, 1991, pp. 459-484 y Sanmarchi, 1999, pp. 509-540. 
inicialmente en potencia, de modo que no puede por sí misma dirigirse hacia su objeto propio, el Bien Universal. Por otra parte, ninguna cosa creada puede mover la voluntad inicialmente al obrar, pues solo Dios, Bien Universal y Perfecto, es capaz de colmar y de inclinar la voluntad de modo suficiente ${ }^{29}$.

En cuanto al segundo momento, la voluntad, a diferencia del primero en el que se muestra pasiva respecto del influjo divino, se manifiesta plenamente activa, pues es propio de la creatura la determinación del acto de elección $(\text { scelta })^{30}$. Ahora bien, Dios no se revela solamente como Causa de las causas, sino también como Causa de los efectos provenientes de las causas causadas, de manera que puede afirmarse que tanto la creatura como Dios son causa directa, inmediata e integral del acto de libertad. La moción divina alcanza la intimidad de todas las acciones sin que nada permanezca ajeno a su infinita causalidad. De allí que Fabro considere que Dios, al causar el esse a todas las creaturas, sea más intrínseco a las cosas que las cosas a sí mismas ${ }^{31}$. En vistas de lo desarrollado, debe tenerse en cuenta que la causalidad total y continua de Dios no excluye la causalidad y libertad de la causa segunda, sino que, por el contrario, la sustenta y fundamenta.

De este modo, al examinar la actividad de la voluntad no debe pasarse por alto la mediación de la inteligencia, pues ambas potencias constituyen, conjuntamente, el acto libre ${ }^{32}$. Tomás de Aquino se pregunta, establecida ya la distinción entre las potencias espirituales, si la voluntad es más elevada que la inteligencia, o a la inversa. En ambos parajes arriba a una idéntica conclusión: simpliciter, la inteligencia es superior a la voluntad; secundum quid, la voluntad es

29 "La volontà come principio finito che passa dalla potenza all'atto, deve anzitutto attuarsi nella tendenza al fine per poter scegliere da se stessa i mezzi; ma non puo attuarsi da se stessa essendo, nel primo momento come creatura, in potenza al fine stesso; perciò bisogna dire che nel primo momento la volontà è mossa al fine solo da Dio stessi" (Fabro, 1955b, p. 133).

30 "En el ámbito existencial, que es el campo de la acción y, por tanto, de la formación del yo y de la persona, el primer principio es la voluntad, cuyo centro dinámico es la libertad" (Fabro, 1981, p. 324).

31 "Se Dio è piu intimo alle cose che non le cose a se stesse, se l'esse è il suo effetto proprio, quiescente e immanente all'ente, allora ciò ch'è più attuoso e presente alle cose è l'esse partecipato, nel senso intensivo e divisivo -come atto dell'essenza-di cui trattiamo" (Fabro, 1969a, p. 117).

32 Inteligencia y voluntad, señala el filósofo italiano, son dos aspectos del yo que el pensamiento moderno los identifica, mientras que el pensamiento clásico los concibe como actividades diversas provenientes de potencias distintas (Fabro, 1986, p. 17). 
superior a la inteligencia. Fabro, como es sabido, muestra profundos reparos a este respecto ${ }^{33}$.

De acuerdo con la exégesis del filósofo italiano, la subordinación de la voluntad a la inteligencia en la actuación de la libertad se presenta de forma férrea en la obra del Aquinate pues, según a Fabro (1983, pp. 22-23), todo acto volitivo -sea en relación con el fin (intentio), sea en relación con los medios (electio)- siempre es entendido como consecuencia de un acto intelectual. La voluntad, en efecto, tiende a su objeto según el orden de la razón, ya que la vis aprehensiva -que en cierto modo antecede a la vis apetitiva - preconcibe el objeto apetecible y lo propone a la voluntad. De allí que Sto. Tomás compare a la inteligencia con la voluntad como principium movens, en cuanto que compete a la potencia intelectual el momento esencial de guía y disposición del acto, y a la potencia volitiva, simplemente, el momento material de la inclinación al objeto como principio agente ${ }^{34}$.

En las Quaestiones Disputatae De Malo Fabro descubre, sin embargo, algunas expresiones del dominico que permiten una profundización de la noción de libertad desde un enfoque más flexible y existencial. En dicha obra el doctor medieval hace la siguiente y fundamental aclaración: si se considera el movimiento de las potencias del alma por parte del objeto que especifica el acto (ex parte obiecti), el primer principio de la moción proviene de la inteligencia. En cambio, si se considera el movimiento de las potencias del alma por parte del ejercicio del acto (ex parte subiecti), el primer principio de la moción proviene de la voluntad (Tomás de Aquino, De Malo, q. 6).

33 En directa relación con este asunto, comenta Danilo Castellano (1984): "Problematica è la collocazione di Fabro fra i neotomisti puri si se considerano le sue tesi strettamente morali, in particolare sull'electio finis ultimi in concreto, vale a dire sulla scelta esistenziale personale della felicità, e sul rapporto volontà-intelletto" (p. 136). Marco Nardone pone también en evidencia la problemática apenas enunciada: "La possibilità di una subordinazione difettiva della volontà all'intelletto non sembra considerata da Fabro, eppure, a nostro giudizio, può rendere ragione della libertà dell'uomo senza rischiare di esporre la electio finis ultimi in concreto a qualche forma di irrazionalità soggetti$v a "$ (Nardone 1999, p. 40).

34 Escribe Fabro (1893) en relación con este tema: "Quello che sorprende allora è l'affermazione categorica: Intellectus comparatur ad voluntatem ut movens, et ideo non oportet distinguere in voluntate agens et possibile $-S$. Th., I, q. 83, a. 4, ad 3-. Un affermazione tutt'altro che evidente e che esige di essere precisata" (p. 63). 
El progreso decisivo de esta argumentación se encuentra en el hecho de que quantum ad exercitium actus, ya no es el objeto aprehendido por la inteligencia el que mueve a la voluntad, sino que es la misma voluntad la que se inclina interiormente. Si la potencia apetitiva espiritual se mueve entonces por sí misma y no por otro (a se ipsa et non ab alio), esto significa que existe un punto de fuerza intacto de libertad en el centro de la voluntad que se sustrae de toda rígida consecutio intentionalis, y que constituye -precisamente como tal- el núcleo profundo de la responsabilidad (Fabro, 1974a, p. 550).

Al tratarse de la potencia a la que incumbe propiamente el fin de todos los fines, la voluntad no solo tiene dominio sobre su propio acto, sino también sobre el acto de todas de las demás potencias, incluida la misma inteligencia, según la célebre expresión tomasiana que tanto place a Fabro: Intelligo quia volo. Efectivamente, el fin y la perfección de todas las potencias del alma se encuentran contenidas bajo el objeto de la potencia volitiva como cierto bien particular, de modo tal que sin la acción primera de la voluntad como motor omnium ninguna potencia podría inclinarse al obrar. Solo la voluntad, subraya el filósofo italiano, se autodetermina originariamente, y esto según una triple valencia del acto: velle (querer), non velle (no querer), nolle (querer no querer). En este sentido, puede hablarse de una emergencia positiva de la voluntad en la estructuración trascendental de la persona como causa suij ${ }^{35}$.

Debe reconocerse a la filosofía moderna, según nota Fabro (1983b, p. 105), la reivindicación del primado activo de la voluntad y la apoteosis de la libertad no como simple propiedad sino como expresión misma de la esencia del espíritu humano. Pese el mérito de dicha empresa, el pensamiento moderno tiene también, como contracara, la eliminación de la trascendencia del ser, principio que -según juzga el autor en cuestión- obstruye el curso auténtico de esta primera y fundamental instancia (Fabro, 1966, p. 584).

35 Lodovici considera que no es correcto afirmar que Fabro desatiende al aspecto subjetivo de oscilación interior, de fragilidad, de conflicto íntimo, que se verifica en el singular y en la historia de vida de cada voluntad (Ruiz Montoya, 2008, p. 279s.), puesto que en sus escritos hay no pocos elementos para sostener lo contrario. No obstante, juzga que el filósofo italiano no ahonda lo suficiente en la incidencia significativa de las virtudes y de los vicios en la elección del bien, como tampoco en el influjo de las pasiones sobre la voluntad y de la voluntad sobre las pasiones (Samek Lodovici, 2012, pp. 191-192). Ambas críticas merecen tomarse en consideración. 
Con Descartes nace la nueva y radical fórmula de la libertad, pues frente al pensamiento clásico que afirmaba la "conciencia del ser", el cogito cartesiano proclama el "ser de conciencia", que representa la autonomía del pensamiento y la superación de toda barrera o límite por parte de la subjetividad. Es cierto, admite el filósofo italiano, que los postulados cartesianos no resuelven todavía el ser en el pensar; sin embargo, al ponerse entre paréntesis -en virtud de la duda metódica- la presencia inmediata y positiva del ser al pensamiento, y afirmarse, en su lugar, la presencia primera y exclusiva del pensamiento a sí mismo, se sientan las bases especulativas para este procedimiento ${ }^{36}$.

Kant avanza por el camino trazado por el filósofo francés ${ }^{37}$ con el anuncio del sujeto trascendental, el cual se despliega en el ámbito teórico mediante el $I c h$ denke como principio de los juicios sintéticos a priori; y en el ámbito práctico, mediante el Sollen como fundamento objetivo de las acciones de la vida moral. En su obra crítica, la libertad es examinada a partir de la consideración de la causalidad de la actividad humana. En efecto, si se miran las acciones del hombre extrínsecamente, es decir, desde el ángulo empírico propio de la experiencia fenoménica, se percibe que están sujetas por entero a las leyes de la causalidad física y mecánica y, por consiguiente, a la fuerza de la necesidad. Sin embargo, si se miran tales acciones intrínsecamente, es decir, en su carácter inteligible, se observa una causalidad nueva y superior, distinta de la ciega y determinista de la naturaleza ligada a las formas puras de la sensibilidad ${ }^{38}$.

En tanto causalidad de la Vernunft, la libertad se presenta entonces con dos sentidos principales: uno negativo, como facultad empíricamente incondicionada; otro positivo, como facultad que comienza por sí una serie de fenómenos. A

36 "É nel pensiero moderno, con il cogito di Cartesio, che compare la formula radicale della libertà, sia quanto al contenuto come quanto alla forma: per questo le altre tappe del pensiero moderno sono da intendere non tanto nel senso di aggiunte o di perfezionamenti, quanto come tentativi di svincolare quella formula iniziale alla sua purezza originaria impedendole di cadere nell'una o nell'altra forma dell'alterità dell'essere che finiva per inceppare e deviare il principio del nuovo cammino" (Fabro, 1960c, p. 131).

37 "Kant è un passaggio obbligato per la comprensione di tutti i problemi dello spirito nel mondo moderno" (Fabro, 1983b, p. 296).

38 Para una visión general de la lectura fabriana del pensamiento kantiano, véase: De Anna, 2012, pp. 213-229. 
este respecto, debe advertirse que para Kant las acciones humanas que se aprecian en el curso de los fenómenos naturales obtienen solamente el efecto de la determinación de la libertad, no así la determinación de la libertad en sí misma. Al encontrarse fuera de la realidad empírica, la voluntad es capaz de dar inicio a una serie de estados fenoménicos sin verse ella misma modificada, esto es, sin tener un comienzo, un antes o después, que la vincule necesariamente con la cadena causal de las condiciones empíricas.

Si la voluntad no sufre ningún tipo de alteración, sino que se comporta al modo de un motor inmóvil ¿Es posible imputar al sujeto la moralidad de sus acciones? Kant responde negativamente, pues el sujeto, dada la imposibilidad que tiene la razón de sobrepasar los datos fenoménicos, se ve impedido de penetrar el giro interior de las demás conciencias, e incluso de tomar conocimiento de la moralidad de su propia conducta ${ }^{39}$. Ahora bien, juzga Fabro, si la voluntad se encuentra por encima de todas las vicisitudes y contaminaciones de lo empíreo, debe negarse al sujeto toda responsabilidad moral de sus actos, no solo por el límite cognoscitivo de la razón sino también, y sobre todo, por la cadencia misma de la libertad, la cual permanece siempre, en virtud de su trascendentalidad, inmutable y sin mancha ${ }^{40}$.

En la filosofía moderna lo trascendental adquiere un significado cada vez más incisivo y categórico ${ }^{41}$. En el idealismo crítico esta noción se encuentra

39 Escribe Kant concretamente (1977): „Die eigentliche Moralität der Handlungen -Verdienst und Schuld-bleibt uns daher, selbst die unseres eigenen Verhaltens, gänzlich verborgen. Unsere Zurechnungen können nur auf den empirischen Charakter bezogen werden. Wie viel aber davon reine Wirkung der Freibeit, wie viel der bloßen Natur und dem unverschuldeten Felder des Temperaments, oder dessen glücklicher Beschaffenheit -merito fortunae- zuzuschreiben sei, kann niemand ergründen, und daher auch nicht nach völliger Gerechtigkeit richten" (p. 505).

40 Para Fabro, el pensamiento kantiano tiene, por esta razón, un trasfondo a-moral: "Ё amorale, perché nella sfera noumenica della ragione non ci possono essere né errori né peccati in quanto essa attua la libertà pura, la quale è librata al di sopra dell'empiria in cui si muovono i singoli: questi allora, in quanto -totalmente- soggetti ai condizionamenti empirici, non cadono piu sotto la imputabilita. Quella, in quanto è librata al di sopra di tali condizionamenti, astrae da ogni inquinamento e perciò sfugge ad ogni imputabilità" (Fabro, 1983, p. 289).

41 "Trascendentale" ha ricevuto nella filosofia un rilievo teoretico sempre più pregnante: da un significato ontico-logico prevalente nella Scolastica, esse è diventato costitutivo della stessa "operazione filosofica" a partire da Kant. Da questo momento il termine "trascendentale" significa ciò ch'è e deve essere dato a priori nel soggetto nel senso di presupposto al sapere, e indica percio la costituzione originaria in senso operante attivo dello spirito e soggetto come anticipante e indipendente e tuttavia determinante, 
todavía cercada por la separación entre lo nouménico y lo fenoménico y por la distinción entre la razón pura y la razón práctica. Para Fabro (1971, p. 15), el idealismo especulativo se aboca a la superación de estas fronteras, abatiendo el muro de la cosa en sí y afirmando el carácter creativo de la libertad.

Fichte desarrolla su doctrina de la ciencia en torno al principio kantiano de la "apercepción trascendental" ${ }^{2}$. Al igual que Kant, sostiene que el Yo es la unidad de la conciencia que aparece como fundamento de todas las representaciones. Ahora bien, mientras que Fichte declara que tal unidad se presenta de forma sintética, Kant afirma que se da de forma analítica. En efecto, el Yo no puede concebirse mediante la conexión de lo múltiple de las representaciones, sino que más bien lo múltiple de las representaciones debe concebirse por medio de la destrucción, difusión y división del Uno sobre lo múltiple mediante la forma de un devenir. El "Yo pienso" que en la obra de Kant se describe como simple acompañante de todas las representaciones, en la de Fichte se convierte en la actividad pura e incondicionada del "Sujeto Absoluto”³. Según Fabro (1964, p. 194), ya no se trata pues de un Yo funcional, sino de un Yo productivo, originario, universal, que no es sustrato, cosa en sí, o substancia, sino que se manifiesta, propiamente, como actividad trascendental.

El cogito cartesiano que en la línea del trascendental kantiano es elevado por el idealismo de Fichte al rango de Absoluto, logra con la dialéctica hegeliana dar cumplimiento al proceso de radicalización de la libertad característico del pensamiento moderno. La noción de libertad expresa, para Hegel, la superación de todas las oposiciones que se presentan en el ámbito de la inmediatez. Esta se identifica con la actividad del Espíritu Absoluto (Fabro, 1967, p. 152). La libertad del individuo, aquella que se distingue frente a un otro, que determina el propio yo, se descubre en el sistema falsa e ilusoria, pues no representa otra cosa más

rispetto all'oggetto di esperienza" (Fabro, 1974b, p. 87). En relación con esta cuestión, véase: Luise, 2012, pp. 329-352.

42 "Forse nessun filosofo moderno, e forse nell'arco del pensiero di tutti i tempi, è stato cosi avvinto dal problema della libertà come Fichte: la tensione eccezionale della sua riflessioni e delle sue polemiche hanno qui il centro, l'oggetto e lo scopo" (Fabro, 1983, p. 133).

43 "Le varie e tormentate Wissenschaftslehren, di cui ha riempito la sua singolare attività speculativa, non sono in realtà che varie Freiheitslehren ossia tentativi di scoprire il fondo e il fondamento della libertà come il nocciolo dell'attività spirituale" (Fabro, 1978, p. 268). 
que aquella libertad de "hacer lo que se quiere" (Fabro, 1954, p. 35). Se trata de una libertad puramente formal y abstracta, de un simple arbitrio que -al permanecer completamente atado a lo útil y contingente- mantiene aún como cosas distintas y separadas el lado objetivo y el lado subjetivo, la inteligencia y la voluntad, el pensar y el querer. El pecado, o bien, la esclavitud del pecado (die Knechtschaft der Sünde) se manifiesta como la pretensión del individuo de ser y de elegir por sí en contraste con el despliegue de la Voluntad Universal que es armonía perfecta de racionalidad e historia ${ }^{44}$.

Ante esta concepción de la libertad, que relega el yo personal al plano de lo puramente transitorio y accidental, que sitúa al sujeto en el terreno de lo inmediato y de la mala infinitud, Kierkegaard coloca -como categoría suprema de la existencia- la interioridad ética-religiosa del "Singular" (Enkelte) $)^{45}$. A este respecto, Fabro trae a colación el famoso enunciado kierkegaardiano que reza "la verdad es la subjetividad" ${ }^{\prime \prime 6}$, pues dicho principio expresa de forma concisa la impugnación que el filósofo danés hace de la metafísica idealista en general y del sistema hegeliano en particular (1995, pp. 76-78).

La existencia, para Kierkegaard, está dada concretamente por el movimiento de la libertad. Esta constituye la esfera del devenir, de la mutación; mientras que la esencia, por el contrario, designa la esfera de lo estable, de lo fijo, de lo necesario. De allí entonces que la existencia no se deje pensar, pues ella elude todo reposo y todo tipo de resultado. En efecto, en el conocer el hombre se encuentra en el campo objetivo de la esencia, y por ende, de la posibilidad (Mulighed); en el obrar, en cambio, se halla en el plano efectivo de la realidad (Virkelighed). Esta, y

44 "L'Io come spirito è la relazione della negatività con se stessa; allora in quanto è relazione a sé è altrettanto indifferente verso questa -cioè verso ogni-determinatezza, la conosce come la sua e ideale, come una pura possibilità, dalla quale non è vincolato ma nella quale esso è, poiché si pone nella medesima. Questa è, per Hegel, la libertà del volere la quale costituisce il suo concetto e sostanzialità, la sua gravità -Schwere-cosi come la gravità costituisce la sostanzialità del corpo" (Fabro, 1983, p. 183).

45 Para un primer acercamiento a la interpretación general de la obra de Kierkegaard por parte de Fabro se recomienda leer Delfgaauw (1984, pp. 457-493).

46 "Quest' incontro fra San Tommaso e Kierkegaard, fra essere e libertà, costituirà l'ambito privilegiato della sua riflessione filosofica. Cosi se l' 'stanza di una fondazione metafisica della libertà ha nel primato dell'essere come atto di San Tommaso il punto di riferimento fondamentale e irrinunciabile, è peró a Kierkegaard e al suo principio che la "verità è la soggettività" che il P. Fabro fa ricorso per rispondere alle istanze piu radicali del pensiero moderno e contemporaneo" (Giannatiempo, 1996, p. 38). 
no aquella, representa - para el danés- la verdadera subjetividad ${ }^{47}$, la cual no se resuelve en el pensamiento puro, en la Razón Absoluta o en la Substancia única, sino que se consolida en sí misma, en su propia singularidad, en tanto principio responsable del actuarse de la propia libertad ${ }^{48}$.

Entre posibilidad y realidad, comenta Fabro (1952, p. 16) siguiendo los textos de Kierkegaard, no hay, propiamente hablando, una relación causal, pues dichas nociones indican dos estados del ser que se distancian tanto uno del otro cuanto se distancia el no-ser del ser. Entre ambas categorías se presenta, por lo tanto, una inconmensurabilidad que se supera solamente a través del salto cualitativo de la libertad. Es menester reparar aquí en el hecho de que para el danés la libertad no tiene vínculo con ninguna cosa precedente, sino solo consigo misma. Tiene, pues, su surgimiento de la nada y es infinita (Kierkegaard, 1995, p. 420). En la reflexión de Kierkegaard, toda consideración de la libertad como mero poder, esto es, como pura posibilidad para la posibilidad, se presenta enteramente formal, puesto que la libertad no se erige de manera absoluta, permaneciendo en una indiferencia vacía, sino con la irrupción radical de la elección.

Para Kierkegaard el hombre es una síntesis entre dos términos: finito e infinito, tiempo y eternidad, posibilidad y necesidad. El yo, sin embargo, no es la relación entre dichos términos, sino la orientación interna de esa relación, es decir, el relacionarse de la relación consigo misma (Kierkegaard, 1995, p. 21). De aquí se sigue, según el pensador danés, que el yo no se presenta realmente libre sino cuando requiere ser sí mismo. Poniéndose en relación consigo mismo se sumerge, a través de su propia transparencia, en la potencia que lo ha puesto. Es

47 "La "verità" è l'intima sostanza della vita dello spirito solo se è interiorità autentica, quella vissuta dal "singolo" nella sua singolarità incomunicabile, nella infinita riflessione di sê" (Fabro, 1945, p. 14). Sobre este tema, véase: Fabro, 1962, pp. 100-108.

48 "Le verità puramente speculative terminano alla contemplazione del loro contenuto astratto: in esso il conoscente riposa. Ma le verità etico-religiosa hanno nel proprio contenuto formale soltanto il punto di partenza, il contenuto "reale" lo hanno nel "termine", nel movimento interiore ed esteriore dell'azione con cui il soggetto si attua in esse ovvero le fa sue: esse esigono di essere "incarnate" nell'azione, nel movimento della libertà. Si può dire che se la prima è verità di Anschauung-Anskuelse-, la seconda è verità di Aneignung -Tilegnelse-; se la prima è inizio, la seconda è fine e compimento; se la prima può essere spesso intuizione di genio e affermazione di differenze individuali, la seconda è possibilità universale offerta all'uomo essenziale, conquista di volontà e fattore primario di personalità" (Fabro, 1955a, p. 70). 
decir, solo con la autoafirmación de sí en el principio, con la elección absoluta de lo Absoluto, el espíritu se determina como espíritu ${ }^{49}$.

Ahora bien, así como la elección de Dios se revela como fundamento existencial de la libertad humana, la omnipotencia divina se presenta para Kierkegaard como su fundamento metafísico, ya que lo más elevado que se puede hacer por un ser es hacerlo libre. Para esto, es necesaria la omnipotencia, pues la potencia finita torna dependiente el obrar de la creatura, en tanto que la omnipotencia lo hace independiente (Kierkegaard, 1962, pp. 512-513). Esto resulta extraño porque la omnipotencia divina debería volver dependiente la acción del hombre. Sin embargo, si se la quiere concebir correctamente, se verá que ella comprende el poder de retomarse a sí misma mientras se dona, de modo que la creatura pueda - por vía de la omnipotencia- ser independiente. Solo Dios puede dar completa y desinteresadamente la libertad a la creatura sin perder un mínimo de poder, más aún, reteniendo precisamente su omnipotencia para que la libertad de la creatura sea posible (Fabro, 1983, pp. 16-17). He aquí, indica el danés, que la omnipotencia divina no solo produce la cosa más imponente de todas, la totalidad del mundo, sino también la más frágil, la libertad del hombre y su independencia respecto de Dios ${ }^{50}$.

Así pues, de acuerdo con la reflexión del filósofo italiano, ser y libertad se corresponden recíprocamente. Son como el cóncavo y el convexo, lo interior y lo exterior, el fondo y el fundamento. Ambas nociones constituyen la dupla dialéctica dentro de la cual surge, se desenvuelve y se cumple la actuación misma del ser del hombre ${ }^{51}$. Una de las interpretaciones más corrientes que aparece sobre el

49 "Anche per Kierkegaard l'io è libertà, ma opera in quanto l'io si riflette nell'Assoluto e questo riflettersi è riferire se stessi, l'oggetto della scelta e la scelta stessa, a Dio. L'io è libero non perché si trasferisce e si annienta nell'Infinito, neppure perché si lascia essere -cioè trascinare all'infinito-nel finito, ma perché si erge come affermazione di capacità di scegliere l'Assoluto" (Fabro, 1983, p. 206).

50 "Kierkegaard esprime questo scaturire originario della libertà dall'onnipotenza divina con una formula di rigore metafisico, non astratto però ma di assoluta esigenza esistenziale e di evidenza maggiore delle prove tradizionali della libertà che si riscontrano nella tradizione cristiana teologica e filosofica -compreso lo stesso San Tommaso e soprattutto la sua scuola-" (Fabro, 1980, p. 124).

51 "Il fondamento è l'essere e il fondo è la libertà: come la libertà attinge il fondamento dell'essere e come l'essere attui il fondo della libertà è cio che propriamente forma il compito costitutivo della filosofia come attuazione dell' istanza fondamentale: - Cosa posso sapere? - Cosa devo fare? - Cosa posso sperare?, per rispondere all'interrogativo finale: Cosa è l'uomo?" (Fabro, 1966-1967, p. 2). 
concepto de libertad se da en sentido negativo, esta resalta la multiplicidad de obstáculos e impedimentos que se presentan a la misma por parte de la naturaleza, la sociedad e incluso el propio yo. Desde esta óptica, la libertad se entiende como el poder de ejercitar, sin restricciones ni ataduras, las virtudes y derechos que le son propios. Anterior a esta libertad aplicada, óntica, regional, se encuentra, empero, una libertad positiva, profunda, radical, que no tiene como fin más que el ser y el hacer de sí, lo que constituye, precisamente, la subjetividad trascendental del espíritu humano ${ }^{52}$.

Esta libertad pura y originaria, señala Fabro, no conlleva una referencia o relación con el objeto, sino que es aquella fuerza primigenia que alimenta la atracción misma del objeto y del fin. La libertad -génesis dinámica de la subjetividad-se presenta así como principio inobjetivable ${ }^{53}$. Esto no significa que esté privada de contenidos que justifiquen su orientación, sino que se encuentra en el momento previo a la objetivación. Anterior al qué, al cómo, al porqué, al dónde, al cuándo, propio de toda tensión de la libertad, está el querer elegir, el querer inclinarse, en definitiva, el querer querer. Efectivamente, toda elección se encuentra precedida por aquel impulso originario del yo que, siendo en sí mismo absolutamente indeterminado, se determina absolutamente a sí mismo ${ }^{54}$.

52 "Per trascendentalità intendiamo quella infinita capacità di originare da se stesso e di riferire tutto il comportamento a se stesso, non alla semplice particolarità spazio-temporale, ma come al centro genetico dell'attuarsi della coscienza" (Fabro, 1969-1970, p. 106).

53 “Ilpensiero è la presenza e il farsi presente dell'essere, è la "presenza del presente", è il porsi davanti e dentro la coscienza, è l'orientare la coscienza rispetto a un contenuto dato epresente. Questo $\dot{e}$ il pensare, che è uno stato quiescente di possesso intenzionale. Volere, scegliere volendo, voler scegliere, invece è il momento dinamico della coscienza: non è il momento della presenza, mal il momento della assenza, quindi della "tendenza a...," della "aspirazione a..., della "passione per..." Questa è la volontà, ch'è appunto la caratteristica ultima e profonda della soggettività stessa dell'io" (Fabro, 1967-1968, p. 96).

54 De allí el carácter de incomunicabilidad propio de la libertad: "La scienza, l'arte, si possono comunicare -un quadro, un pezzo musicale, una poesia-... allora c'è una specie di presenza che si intensifica, c'è quel godimento estetico, quel dilatarsi interiore e riversarsi l'uno nell' altro, quell'entusiasmarsi che è proprio della sfera estetica, della sfera immediata ecc... La libertà non è delegabile -io non posso farvi liberi, voi non potete farmi libero, voi non potete darmi la libertà e neanche togliermela [...]-. La libertà interiore è quella che dà significato, è quella che colorisce penetrando fibra a fibra il mio essere, e dà senso alle mie scelte, alle mie azioni, ai miei amori. È l'amore di tutti gli amori, la forza di tutte le forze: questa è la libertà!"'(Fabro, 2007, pp. 77-78). 
La libertad se autocualifica, se autoestructura, se autoespecifica, según su propio deseo y aspiración, ya que la voluntad posee en su interior el poder de querer el bien y de querer el mal. Comúnmente se dice -sobre todo en el campo de la filosofía moral- que cuando se quiere el mal no se quiere por sí mismo, sino bajo la apariencia o aspecto de bien. En el plano existencial, sin embargo, las cosas se presentan de manera distinta, ya que se percibe que no solo la elección del bien es una determinación de la voluntad, sino también la elección del mal, por reprobable que ella sea. Todavía más, indica Fabro (1967-1968, p. 95), en esta esfera se advierte que la elección del bien, si no tuviera contra sí la posibilidad misma de la elección del mal, no emergería nunca como tal.

En numerosas ocasiones se ha concebido al mal como mera falta, carencia, defecto, privación, todas notas que bajo uno u otro aspecto indican la confinación de su realidad al no-ser ${ }^{55}$. El filósofo italiano, en determinados casos, observa que la elección del mal reclama un ímpetu mayor que la elección del bien. De modo que no puede afirmarse que el mal sea efecto o resultado de la debilidad del sujeto, sino que más bien que se trata de un acto tan vehemente y positivo como el de la elección del bien ${ }^{56}$. Incluso, declara Fabro, puede decirse que la positividad del mal es más constitutiva - por paradójico que parezca- que la positividad del bien, porque cuando se hace el bien se produce una cierta inserción trascendental del individuo en el principio, una especie de concurso y participación del yo en la causa suprema, una unión con el Absoluto que trasciende los límites de la subjetividad. En cambio, cuando se hace el mal se advierte la propia especificidad, es decir,

55 "Dolore, sofferenza, ignoranza, perversione, vizio, disperazione, insuccessi, incomprensioni, fallimenti... e morte formano l'orizzonte di quel che l'umanità ha chiamato, e anzitutto sperimentato, come "il problema del male." È un problema che di solito resta alla tangente, almeno nella tradizione filosofica occidentale, la quale preferisce partire dall'essere e dal bene per orientare subito sulla coscienza e sulla prassi, per vivere e godere la vita e solo in un secondo tempo, quando la vita s'incespica nei suoi labirinti, essa avverte il male e si cruccia per l'ostacolo, si dispera per il dolore" (Fabro, 1988, p. 3).

56 Lo que se afirma respecto de la realidad del mal puede decirse para Fabro de igual manera en relación con el pecado: "En la concepción cristiana del pecado, su esencia consiste en ser un acto de "desobediencia" del hombre a la ley de Dios y, por tanto, de "rebelión" de la voluntad creada a la voluntad de Dios; es esta deformidad ontológica de la voluntad del hombre respecto a la voluntad de Dios la que confiere al acto humano su deformidad moral. El pecado no se resuelve por ello en una simple privación, como ser ciego o cojo, sino que podría decirse que es una "privación activa" porque el pecado lleva consigo un acto por parte del hombre, una alternativa del bien y del mal que compromete al hombre en y para el mal conscientemente. Por eso se habla de responsabilidad y también de imputabilidad" (Fabro, 1963a, p. 132). 
el individuo percibe -en su insubordinación contra Dios- su autoconstitución y diferencialidad (Fabro, 1967-1968, p. 102).

Bien y mal, por lo tanto, no son una alternativa lógica como la que se despliega entre el sí y el no, entre la afirmación y la negación, entre la presencia y la ausencia, sino que es una disyuntiva radical en la cual deviene, se cualifica y se posiciona la libertad. La voluntad, en efecto, no es un principio pasivo, sino máximamente activo y libérrimo, de modo que en sí reside la raíz y fundamento del bien y del mal. Lejos de ser algo neutral, una facultas ad utrumlibet, la voluntad se manifiesta como fuerza propulsiva, pasión refleja, empeño esencial ${ }^{57}$.

Para Fabro, la libertad en sí misma no admite ningún tipo de escalafón, no es buena ni mala, no tiene un más ni un menos, sino que se revela metafísicamente idéntica en todos los sujetos. Efectivamente, su cualidad es ser sin cualidad, su valor es ser sin valor, su carácter es ser sin carácter, pues la libertad se da a sí misma, libremente, su propia cualidad, su propio valor, su propio carácter. No puede afirmarse, por consiguiente, que en ella existan grados ni jerarquías; la libertad siempre se presenta de un modo infinito y absoluto. En este sentido, señala el filósofo italiano, la libertad del hombre no es inferior a la libertad divina, sino de idéntica naturaleza y perfección, puesto que si bien la libertad del hombre depende de Dios, dicha dependencia es del todo peculiar, pues la voluntad del hombre es capaz de ir contra la de Dios, aun cuando Este habita en su ser por esencia, presencia y potencia ${ }^{58}$.

\section{Conclusión}

YA eXPUeSTO, SUCINTAMENTE, los conceptos y principios más importantes de la obra de Fabro, es momento de dar cierre a este trabajo con algunas breves consideraciones. En opinión del filósofo italiano, la libertad es la energía primordial a la que todos los actos y planos de la conciencia se remiten, no solo el ámbito de

57 Este punto, precisamente, es el que opera en el fondo de aquella tesis tan conocida de Fabro de la elección existencial del fin último en concreto. En cuanto a esta controvertida cuestión, véase: García-Baliño Abós, 2010.

58 "L'uomo si eleva a spirito e gode della libertà piena, suprema, assoluta... la quale come qualità non differisce e non può differire da quella di Dio, e questo si prova dal fatto che l'uomo può tanto scegliere come rifiutare Dio" (Fabro, 1968b, p. 18). 
la sensibilidad y del intelecto, sino también de la razón, pues si la libertad se encontrara condicionada por esta última acaecería como mera conclusión ${ }^{59}$. Sin embargo, para Fabro la libertad no es una consecuencia sino el principio de todo, la razón de la razón, porque esta no podría discurrir, avanzar, examinar, si no se hallara previamente este movimiento primigenio que no se dirige hacia esto o aquello, por esto o por aquello, sino que es la capacidad de mover el moverse mismo, del mover a sí mismo, del liberarse a libertad ${ }^{60}$.

Entre la libertad y la voluntad hay una autoappartenenza originaria ${ }^{61}$, pues la voluntad es su principio próximo e inmediato, en tanto que la espiritualidad del alma se revela como su fundamento último o remoto. Los filósofos modernos, en sentir del filósofo italiano, tienen el logro de haber evidenciado la principalidad del yo en la compleja labor de su deducción trascendental; no obstante, al preocuparse por justificar únicamente el carácter suprasensible de la actividad de la conciencia, dejaron de lado el núcleo que conduce a la verdadera realización de la libertad, a saber: el acto de elección. De este modo, llevaron adelante, progresivamente, una supresión de la consistencia ontológica del individuo como ser en sí y centro genético operativo. Las acciones del hombre (deliberación, decisión, entre otras) no recaen, desde esta perspectiva, en la autointuición del yo individual, sino en la intuición de la Egoidad ${ }^{62}$.

59 "Questo primato dinamico della volontà non è però di pura efficienza, ma il fine scelto investe tutta la sfera esistenziale ossia, "informa", per cosi dire, l'attività intera del soggetto come persona, dall'intelligenza fino alle altre facoltà appetitive e conoscitive in una specie di circulatio libertatis ch'è una partecipazione dinamica, ma insieme un'assunzione di solidarietà e responsabilità dell'agire da parte della persona come un tutto, guidato bensi dall'intelligenza -più o meno secondo i casi- ma mosso e dominato dalla volontä" (Fabro, 1893, p. 65).

60 “La ragione prepara l'atto volontario come l'occhio prepara l'atto d'amore per la bella figura. Dunque tutto dipende dall'occhio? No, tutto dipende dalla vaghezza, dal fascino, dall'attrazione dell'amore. Ecco perché anche per S. Tommaso la libertà è il nocciolo più profondo della vita dello spirito: perché l'oggetto della volontà e quindi della libertà è il bene, è la felicità ed il bene e la felicità è l'oggetto di tutta la persona. [...]. La volontà è la facoltà totale della persona totale, non cosi l'intelligenza o la sensibilità. L'intelligenza e la sensibilità variano da tipo a tipo; l'intelligenza coglie gli aspetti dirimenti del reale, invece l'amore unisce, l'amore organizza tutta la persona" (Fabro, 1972, p. 180).

61 En cuanto a la opción de Fabro por investigar la libertad humana a partir de la voluntad y la importancia que tiene esta perspectiva en el debate contemporáneo sobre la autonomía personal, véase: Giovagnoli, 2012, pp. 427-440.

62 Y sin embargo, señala el filósofo italiano, no hay realidad más alta, más elevada, más excelsa después de Dios, que la de mi propio yo. Ver: Fabro, 2000, $\$ 842$. 
Frente a esta concepción filosófica de carácter despersonalizante, Fabro declara que hic homo singularis eligit, tendit, amat ${ }^{63}$. Inspirado en los escritos kierkegaadianos, el filósofo italiano confiere al yo del singular, al Io volo, el carácter de "Absoluto" que los sistemas especulativos de raigambre spinoziano conceden propiamente al Yo universal. El hombre, ser finito, limitado, dependiente desde el punto de vista metafísico, se manifiesta independiente, absoluto y autónomo en la esfera existencial ${ }^{64}$. De allí que la libertad sea definida por Fabro como incondicionalidad condicionada, independencia derivada, es decir, absolutez por participación. Solo en Dios, Ser por esencia, su libertad es libertad por esencia. Así, como en el plano metafísico puede decirse que operari sequitur esse, en el plano existencial puede afirmarse que esse sequitur operari. En efecto, la libertad tiene el poder de hacer que sea y que devenga lo que de otro no hubiera sido ni devenido, y a la inversa, de hacer que no sea y que no devenga aquello que de otro modo hubiera sido y devenido. En esto se manifiesta justamente el carácter creador de la libertad ${ }^{65}$.

En el pensamiento de Fabro, tal como se ha procurado evidenciar, se encuentran distinguidos cuidadosamente dos órdenes: el metafísico y el existencial. En el primero, el acto de ser se revela como acto de todos los actos. En el segundo, la libertad se revela como acto de todos los actos. En efecto, la existencia no es otra cosa - para el filósofo italiano- que la libertad misma en acto (Fabro, 1983a, p. 23). Así pues, a este respecto es menester advertir que Fabro expone un estudio claro y profundo sobre la cuestión del ser. Asimismo, despliega un análisis agudo sobre el problema de la libertad; sin embargo, cabe preguntarse si examina, con el mismo detenimiento y sistematicidad, la relación esencial que se establece,

$63 \mathrm{Al}$ igual que Tomás de Aquino acentúa, contra el mono-psiquismo averroísta, que hic homo singularis intelligit, Fabro reivindica - por su parte- la consistencia del sujeto espiritual a través del principio que tiene el dominio y responsabilidad de la actividad de la persona entera. (Fabro, 1983c, pp. 153-154).

64 "L'ente spirituale -uomo, angelo- si pone su due piani: a) in quello ontico-metafisico dell'essere ed è una creatura soggetto alla causalità di Dio o realtà causata e finita nel suo essere ed operare che sono dipendenti, b) in quello ontologico-esistenziale dell'agire cioè della sua attività spirituale ch'è libera e indipendente" (Fabro, 1975. p. 530).

65 "La libertà è precisamente quella capacità dell'uomo, e dell'uomo soltanto, "mediante" la quale qualcosa puo essere che altrimenti non sarebbe e qualcosa non puo essere che altrimenti potrebbe essere" (Fabro, 1969, p. 8). 
forzosamente, entre dichos actos y órdenes. Según mi apreciación personal, el filósofo italiano no logra alcanzar una síntesis suficientemente armónica, porque si bien es cierto que en su obra aparecen valiosas indicaciones, sugerencias y anotaciones sobre este asunto, estas se encuentran diseminadas por doquier, sin un tratamiento ordenado ni articulado. Parecería tratarse de intuiciones aisladas que no obtienen, posteriormente, un mayor desarrollo ${ }^{66}$.

Probablemente la separación o hyatus entre el plano metafísico y el existencial no sea sino la consecuencia inevitable de la antítesis misma que se presenta entre dos instancias teoréticas de suyo divergentes: por un lado, la tomasiana del esse ut actus; por otro lado, la moderna -fundamentalmente idealista- de la $l i$ bertas ut actus ${ }^{67}$. Si este hecho se corroborara, debería entonces afirmarse que la esfera metafísica y la esfera existencial no representan solamente dos perspectivas de análisis diferentes sino, antes bien, dos concepciones metafísicas distintas. Tal aserción, sin embargo, reclama ulteriores especificaciones que abren el tema a futuras investigaciones.

No compete decir aquí si Tomás de Aquino entiende o no al actus essendi en sentido activo, pero sí puede aseverarse que para el filósofo italiano el ser no se identifica con la activitas. En efecto, Fabro concibe al esse como aquello que permanece inmóvil en el fondo de las cosas, o bien como escribe el mismo Aquinate, como aquello que reposa de modo fijo y quieto en el ente ${ }^{68}$. De aquí se sigue la distinción entre el orden estático y el orden dinámico, entre el ser de primer

66 No parece convincente, en este sentido, la posición de Christian Ferraro, quien declara en la conclusión de su trabajo: "Es lícito hablar, por consiguiente, de dos comienzos, uno metafísico formal y otro metafísico existencial: el primero es el de la reflexión especulativa y el segundo el de la realización existencial. El primero mira al ens sicut primum formale, mientras que el segundo mira al Yo que por la libertad se constituye en primum existentiale: es lo que Santo Tomás indicaba como motus ex parte subiecti, cuyo principio inmediato instrumental es la voluntad, y cuya sólida fórmula especulativa es el intelligo quia volo" (Ferraro, 2009, pp. 501-502).

67 Elvio Fontana parecería ver en la obra de Fabro, por el contrario, una fidelidad total al pensamiento de Tomás de Aquino, incluso en su novedad especulativa: "Il pensiero di Fabro è un autentico tomismo perché ha rispolverato la metafisica dell'atto ed è un tomismo moderno, modernizzato, aggiornato, un tomismo ringiovanito perché ha tentato di fondare i principi della metafisica dell'atto e trarre le conseguenze. Ritengo che questi principi essenziali sono stati attivati principalmente grazie alla finezza intellettuale di Fabro" (Fontana, 2007, p. 8).

68 "Esse autem est aliquid fixum et quietum in ente" (Tomás de Aquino, Summa Contra Gentiles, lib. 1, c. 20). 
grado y el ser de segundo grado, entre el ámbito formal y el ámbito real. Más allá de la serie de dualismos que aparecen en la obra del filósofo italiano, debe tenerse cuidado de simplificar las cosas, pues la noción de libertad no recibe en los escritos de Fabro un tratamiento unívoco. Basta con mencionar que el autor, en algunos fragmentos de su obra, sostiene una identidad de tipo dinámico entre el alma y sus potencias, entre el yo y la voluntad, entre el ser y la libertad. Tal asunto, precisamente, es el que ha dado pie a los estudiosos y expertos a diversas - cuando no contrapuestas- interpretaciones sobre la posición de Fabro respecto del concepto de libertad ${ }^{69}$.

La indagación especulativa del filósofo italiano no debe ocultarse, no está libre de vacilaciones, incertidumbres y ambigüedades. Se trata de un pensador enérgico, dinámico, que se replantea las tesis, que vuelve sobre sus principios, que reformula sus conclusiones. Cornelio Fabro no se ha contentado con la mera enunciación de fórmulas que otros pensadores han defendido profusamente, sino que se ha sumido en una reflexión filosófica "vital" que ha interpelado desde lo hondo su propio ser, existencia y libertad.

\section{Referencias}

Abbagnano, N. (1990). Ricordi di un fllosofo. Milano: Rizzoli.

Acerbi, A. (2005). La libertà in Cornelio Fabro. Roma: Edizioni Università della Santa Croce.

Acerbi, A. (2012). Fabro e l'assimilazione metafisica dell'esistenzialismo. En: Aa.Vv. Crisi e destino della filosofia. Studi su Cornelio Fabro. Roma: Edusc.

69 Ariberto Acerbi sostiene en su tesis doctoral una opinión semejante a la vertida aquí, aunque no se pronuncia concretamente sobre la posibilidad de hallazgo de conceptos metafísicos heterogéneos y contrastantes en la obra del filósofo italiano: "La mancanza di tale sintesi di essere e libertà, di cui pure sono apprestati tutti gli elementi, ci pare essere all'origine della distinzione un po' troppo neta da parte di Fabro tra la prospettiva metafisica e la prospettiva esistenziale. Tale distinzione è da lui sistematicamente utilizzata, sempre presupposta, talvolta illustrata, ma mai chiaramente spiegata" (Acerbi, 2005, pp. 252-253). En un trabajo posterior, el especialista revalida su impresión al respecto: "Nell'opera di Fabro si nota infine la mancanza di una elaborazione metafisica positiva della libertà, sebbene nei suoi scritti si presti attenzione agli spunti rinvenibili in tal senso o alle caratterizzazione fenomenologiche dell'io e dell'volere eventualmente suscettibili di un simile approfondimento" (Acerbi, 2012, p. 417). 
Binetti, M. (2003). El itinerario de la libertad. Un estudio basado en el Diario de Soren Kierkegaard según la interpretación de Cornelio Fabro. Buenos Aires: Ciafic.

Borghessi, M. (2012). Essistenza e libertà in Fabro. L'istanza positiva del moderno. En: Aa. Vv. Crisi e destino della filosofia. Studi su Cornelio Fabro. Roma: Edusc.

Castellano, D. (1984). La libertà soggettiva. Cornelio Fabro oltre moderno e antimoderno. Napoli: E.S.I.

Da Silveira, C. (2006). Cornelio Fabro intérprete de Santo Tomás. Aquinate, 3 , pp 1-14.

Dalledonne, A. (2001). Cornelio Fabro. Essere e libertà come fondamenti del tomismo essenziale. Milano: Seam.

Davini, S. (2004). Cornelio Fabro and the Reception of Philosophical Fragments. Kierkegaard Studies 9, pp. 356-369

De Anna, G. (2012a). Fabro interprete di Kant. Libertà trascendentale, amoralità e ateismo. En: Aa. Vv., Crisi e destino della filosofia. Studi su Cornelio Fabro. Roma: Edusc.

De Anna, G. (2012b). Introduzione. En: Aa. Vv. Verità e libertà. Saggi sulpensiero di Cornelio Fabro (pp. 7-12). Napoli: E.S.I.

Delfgaauw, B. (1984). Il contributo agli studi kierkegaardiani. En: Aa.Vv. Essere e Libertà. Studi in onore di Cornelio Fabro (pp. 457-493.). Rimini: Maggioli.

Fabro, C. (1893). La dialettica d'intelligenza e volontá nella costituzione dell'atto libero. En: Riflessioni sulla libertà (pp. 57-85). Rimini: Maggioli.

Fabro, C. (1945). Problemi dellesistenzialismo (1 ${ }^{a}$ ed.). Roma: A.V.E.

Fabro, C. (1952). Antologia Kierkegaardiana. Torino: S.E.I.

Fabro, C. (1954). L'Assoluto nell'esistenzialismo. Catania: Guido Miano.

Fabro, C. (1955a). Attualità è ambiguità dell’opera Kierkegaardiana. En: Sonderdr. Symposium Kierkegaardianum (pp. 66-74). Copenhaghen.

Fabro, C. (1955b). Lanima. Introduzione al problema dell'uomo. Roma: Studium.

Fabro, C. (1960a). Breve introduzione al Tomismo. Roma: Desclée.

Fabro, C. (1960b). Libertà ed esistenza. Thomistica morum principia, 1, pp. 323-330. 
Fabro C. (1960c). Pensiero Moderno e Cristianesimo. En: Filosofia e formazione ecclesiastica. Città del Vaticano.

Fabro, C. (1961). Partecipazione e Causalità secondo san Tommaso d'Aquino (1 ed.). Torino: S.E.I.

Fabro, C. (1962). Introduzione al Diario di S. Kierkegaard. En: S. Kierkegaard, Diario, vol. 1 (C. Fabro, trad.) (2a ed. revisada). Brescia: Morcelliana.

Fabro, C. (1963a). El problema del pecado en el existencialismo. En: Enciclopedia de la ética y moral cristianas: el pecado en la modernidad (vol. 13). Madrid: Rialp.

Fabro, C. (1963b). Il trascendentale esistenziale e il fondamento della libertà. En: Riflessioni sulla libertà (pp. 87-132). Rimini: Maggioli.

Fabro, C. (1963c). L'uomo moderno e la mediazione dell'essere. En: El problema del hombre (pp. 7-14-). México: U.N.A.M.

Fabro, C. (1963d). La nozione metafisica di partecipazione secondo S. Tommaso d'Aquino. Torino: S.E.I.

Fabro, C. (1964). Ateismo e cristianesimo come momenti risolutivi del pensiero moderno. En: Il mondo di Domani (pp. 189-223). Roma: Abete.

Fabro, C. (1965). Dall'essere allesistente (2a ed.). Brescia: Morcelliana.

Fabro, C. (1966-1967). Essere e Verità. Il ritorno al fondamento. Dispensa pro manuscripto. Perugia: Università di Perugia.

Fabro, C. (1966). La libertad en el pensamiento contemporáneo (Trad. E. Benedicto). Atlántida, 24, p. 584.

Fabro, C. (1967-1968). Essere e Libertà. Dispensa pro manuscripto. Perugia: Università di Perugia.

Fabro, C. (1967). Kierkegaard e San Tommaso. Mater Ecclesiae, 3, pp. 152-160.

Fabro, C. (1968a). Genesi storica dell'ateismo contemporaneo. En: Láteismo contemporaneo. Torino: S.E.I.

Fabro, C. (1968b). Libertà ed esistenza nella filosofia contemporanea. En: Annuario dell'Università degli studi di Perugia (pp. 45-65). Perugia.

Fabro, C. (1969a). Tomismo epensiero moderno. Roma: P.U.L.

Fabro, C. (1969b). Elementi per una dottrina tomistica della partecipazione. En: Esegesi tomistica (pp. 421-448). Roma: P.U.L. 
Fabro, C. (1969c). Introduzione all'ateismo moderno. Vol. 1. Roma: Studium.

Fabro, C. (1969). San Tommaso e la libertà. En: Losservatore romano, 7 de marzo, p. 3 .

Fabro, C. (1971). La trascendentalità nella dialettica hegeliana. En: Tempo e storicità dell'uomo (pp. 11-46). Padova: Gregoriana.

Fabro, C. (1972). La libertà in Hegel e S. Tommaso. Sacra doctrina, 66, pp. 165-186.

Fabro, C. (1974a). Freedom and Existence in Contemporary Philosophy and in St. Thomas. The Thomist, 38, pp. 521-556.

Fabro, C. (1974b). La svolta antropologica di Karl Rahner. Milano: Rusconi.

Fabro, C. (1975). La libertà in San Bonaventura. En: Atti del Congressso Internazionale per il VII centenario di S. Bonaventura da Bagnoregio, Miscellanea Francescana, pp. 507-535.

Fabro, C. (1978). Breve discorso sulla libertà. Annotazioni su Fichte. Rivista di Filosofia Neoscolastica, 70, pp. 267-280.

Fabro, C. (1980) Dialettica di libertà-necessità nella storia in Tolstoj e Kierkegaard. En: Tolstoj oggi (pp. 111-128). Firenze: Sansoni.

Fabro, C. (1981). El primado existencial de la libertad. Scripta Theologica, 13, pp. 323-337.

Fabro, C. (1983a) La preghiera nel pensiero moderno. Roma: Storia e Letteratura.

Fabro, C. (1983b). Ateismo e deviazione radicale della libertà. En: Riflessioni sulla libertà (pp. 271-314). Rimini: Maggioli.

Fabro, C. (1983c). Atto esistenziale e impegno della libertà. Divus Thomas, 86, pp. 153-154

Fabro, C. (1983d). La dialettica. Antologia Sistematica. Brescia: La Scuola.

Fabro, C. (1986). Libertad y persona en Santo Tomás. Gladius, 2, pp. 5-32.

Fabro, C. (1988). Il mistero del male. L'osservatore romano, 21 febbraio.

Fabro, C. (1995). Introduzione all'opera di S. Kierkegaard. En: S. Kierkegard, Opere (pp. 25-143). Vol. 1, (Trad. C. Fabro). Casale Monferrato: Piemme.

Fabro, C. (1997). Introduzione a San Tommaso. La metafisica tomista e il pensiero moderno. Milano: Ares.

Fabro, C. (2004). La prima riforma della dialettica hegeliana. Segni: Edivi.

Fabro, C. (2007). La crisi della ragione nel pensiero moderno. Udine: Forum. 
Ferraro, C. (2009). Creatividad participada. La interpretación de la libertad radical según el tomismo essencial de Cornelio Fabro Tesis de doctorado. Pontificia Università Lateranense. (Sin publicar).

Fontana, E. (2007). Attualità del tomismo di Cornelio Fabro. Tesis de doctorado. Pontificia Studiorum Universitas a Sancto Thoma Aquinate. (Sin publicar).

García-Baliño, A. (2010). La voluntad humana en Tomás de Aquino. Un estudio desde sus fuentes griegas, patrísticas y escolásticas. Tesis de doctorado. Universidad de Málaga. (Sin publicar).

Giannatiempo, A. (1996). P. Cornelio Fabro e il suo tempo: la verità inattuale. En: Aa.Vv. Cornelio Fabro. Ricordi e testimonianze (pp. 33-43). Potenza: Ermes.

Giovagnoli, R. (2012). A partire da Cornelio Fabro: libertà, autenticità e autonomía. Aquinas, 55, pp. 427-440

Goglia, R. (2010). Cornelio Fabro. Profilo Biografico, cronologico, tematico, da inediti, note di archivio, testimonianze. Segni: Edivi.

Kant, I. (1977). Kritik der reinen Vernunft. En: Weischedel (Hrsg.) Werke in zwölf Bänden, Bd. 3. Frankfurt am Main: Suhrkamp. Werke in zwölf Bänden, Bd. 3. W. Frankfurt am Main: Suhrkamp.

Kierkegaard, S. (1962). Diario (Trad. C. Fabro). Brescia: Morcelliana.

Kierkegaard, S. (1995). Il concetto dell'angoscia. En: Opere (pp. 305-474). Vol. 1 (Trad. C. Fabro). Casale Monferrato: Piemme.

Kierkegaard, S. (1995). La malattia mortale. En: Opere (pp-13-149). Vol. 3 (Trad. C. Fabro). Casale Monferrato: Piemme.

Knappik, F. (2012). Logica e libertà in Hegel e Cornelio Fabro. En: Aa.Vv. Crisi e destino della filosofia. Studi su Cornelio Fabro (pp. 269-287). Roma: Edusc.

Lobato, A. (1996). El P. C. Fabro, discípulo de Tomás de Aquino. En: Aa.Vv. Cornelio Fabro. Ricordi e testimonianze (pp. 50-54). Potenza: Ermes.

Luise, G. (2012). Trascendentale e trascendenza. Joseph Maréchal e Cornelio Fabro interpreti di Kant. En: Aa.Vv. Crisi e destino della filosofia. Studi su Cornelio Fabro (pp. 329-352). Roma: Edusc.

Mondin, B. (2000). Essenza. En: Dizionario Enciclopedico del pensiero di san Tommaso d'Aquino (pp. 260-268). Bologna: Studio Domenicano. 
Nardone, M. (1999). Cornelio Fabro: il desiderio di Dio di fronte al rischio della libertà. En: Aa.Vv. Per Cornelio Fabro. Cinque saggi in memoria (pp. 23-44). Udine: La Nuova Base.

Negro, M. (2009). Per una critica della dialettica e del nichilismo: la filosofia e il problema del cominciamento in Fabro. Acta Philosophica: rivista internazionale di Filosofia, 18, pp. 363-380.

Olivera, J. (2007). El punto de partida de la metafísica de Santo Tomás de Aquino, según Cornelio Fabro. Tesis de doctorado. Pontificia Università Lateranense. (Sin publicar).

Pandolfi, C. (2006). La distinzione reale tra essentia ed esse in Cornelio Fabro. En: Neotomismo e Suarezismo. Il confronto di Cornelio Fabro (pp. 137-152). Roma: A.P.R.A.

Pangallo, M. (1991). Il principio di causalità nella metafisica di S. Tommaso. Saggio di ontologia tomista alla luce dellinterpretazione di Cornelio Fabro. Città del Vaticano: Pontificia Accademia di S. Tommaso.

Pellecchia, P. (1991). La valenza critica della partecipazione nell'opera di C. Fabro. Aquinas, 34, pp. 459-484.

Pizzuti, G. (2000). Introduzione: quasi un breviario di filosofia. En: C. Fabro, Libro dell'esistenza e della libertà vagabonda (pp. 9-16). Casale Monferrato: Piemme.

Rizzacasa, A. (1997). Cornelio Fabro interprete di Kierkegaard. Euntes Docete, 50, pp. 261-283.

Ruiz Montoya, C. (2008). La Existencia como novedad en Cornelio Fabro. Un análisis de la existencia en clave metafísica. Roma: Edizioni Santa Croce.

Samek Lodovici, G. (2012). Intelligo quia volo. La dialettica di intelletto e volontà in Fabro. En: Aa.Vv. Crisi e destino della filosofia. Studi su Cornelio Fabro (pp. 173-194). Roma: Edusc.

Sánchez Sorondo, M. (2007). Circolarità fra libertà ed essere assoluto per partecipazione in Cornelio Fabro. En: Aa.Vv. Cornelio Fabro e il problema della libertà (pp. 43-66). Udine: Forum.

Sanmarchi, A. (1999). Teoresi essenziale del principio di causalità nel confronto fra san Tommaso e il neotomismo. Rivista di Filosofia Neoscolastica, 4, pp. 509-540. 
Tomás de Aquino. (2007). Summa Contra Gentiles. (Trad. J.M. Pla Castellano). Madrid: B.A.C.

Vijgen, J. (2005) The Future of Cornelio Fabro's Legacy. Doctor Angelicus, 5, p. 199.

Villagrasa, J. (2001). La Gestalt metafisica di Tommaso d'Aquino secondo Cornelio Fabro. Alpha Omega, 14, pp. 416-418.

Wippel, J. (2012). Fabro: sulla distinzione e composizione di essenza ed esse nella metafísica di Tommaso d'Aquino. En: Aa.Vv., Crisi e destino della filosofia. Studi su Cornelio Fabro (pp. 139-156). Roma: Edusc. 